J. Clin. Chem. Clin. Biochem.

Vol. 17, 1979, pp. 9-13

\title{
Kreatinkinase und Kreatinkinase-Isoenzym MB: Erfahrungen mit der neuen Optimierten Methode mit N-Acetylcystein als Aktivator bei Gesunden und bei akutem Myokardinfarkt
}

\author{
Von Ernst W. Schmidt und W. Bender \\ Institut f. Laboratoriumsmedizin, Stadtkrankenhaus Rüsselsheim
}

(Eingegangen am 11. Mai/14. August 1978)

\begin{abstract}
Zusammenfassung: Es wird über erste Ergebnisse mit der N-Acetylcystein-aktivierten Kreatinkinase und ihrem Isoenzym MB bei Gesunden und bei Patienten mit akutem, transmuralem Myokardinfarkt berichtet. Bei Gesunden betrug die obere Normgrenze der Kreatinkinaseaktivität 54 U/1, die Entscheidungsgrenze für das Vorliegen einer Kreatinkinase-MB-Aktivität lag bei 9 U/l. Den nur wenig differenten Grenzwerten im Vergleich zur GSH-aktivierten Kreatinkinase entspricht ein linearer Zusammenhang mit einem Steigungsfaktor nahe 1,0 zwischen den Ergebnissen mit den alten und den neuen Methoden. Die unterschiedliche Reaktivierbarkeit von Kreatinkinase-MM durch GSH und NAcetylcystein wird diskutiert; eine Umrechnung der Aktivitäten von Kreatinkinase und Kreatinkinase-MB von der GSH-aktivierten Methode in die N-Acetylcystein-aktivierte Methode ist nicht möglich.
\end{abstract}

Creatine kinase and creatine kinase isoenzyme MB: Determination of normal values and values for myocardial infarct, using the new, optimized method with $N$-acetyl cysteine as the activator

Summary: Preliminary results are reported for the determination of creatine kinase and its isoenzyme MB in healthy individuals and in patients with acute, transmural myocardial infarct, using $\mathrm{N}$-acetyl cysteine as the activator of the enzyme. In healthy individuals, the upper normal limit for creatine kinase activity was $54 \mathrm{U} / 1$; the decision level for the presence of creatine kinase MB activity $9 \mathrm{U} / 1$. The limiting values were only slightly different from those for the GSH-activated creatine kinase, and the results with the old and new methods showed a linear relationship with a slope of 1.0. The differences in the activation of creatine kinase MM by GSH and by $\mathrm{N}$-acetyl cysteine are discussed; the activities obtained for creatine kinase and creatine kinase-MB by the GSH-activation method cannot simply be recalculated to give the appropriate values for the $\mathrm{N}$-acetyl cysteine activation method.

\section{Einleitung}

Die Messung der Kreatinkinase (ATP: Kreatin-N-Phosphotransferase; EC 2.7.3.2) kann durch verschiedene Einflüsse gestört weŕden $(1-5)$. Fehlbestimmungen durch die Anwesenheit von Adenylatkinase (ATP: AMP-Phosphotransferase; EC 2.7.4.3) aus Lebergewebe oder Erythrocyten und durch Glutathionreduktase (EC 1.6.4.2) kommen am häufigsten vor. Durch Verwendung von N-Acetylcystein als Aktivator $(3,6)$ und durch Zusatz einer größeren Menge von AMP sowie von Diadenosin-pentaphosphat $(6,7)$ wirde versucht, dièse Ștörungen zu beseitigen $(6,7)$.

Da bei der Messung der Aktivität des Kreatinkinase-Isoenzyms MB mittels Immuninhibition $(7-13)$ durch Adenylatkinase- und/oder Glutathionreduktaseaktivitäten im Serum das Ergebnis besonders nachhaltig verfäischt wer- den kann (5), entspricht die Einführung der neuen, optimierten Reaktionsbedingungen bei dem KreatinkinaseMB-Test ${ }^{1}$ ) einem dringenden Bedürnis. In der vorliegenden Arbeit wird über erste Ergebnisse mit der N-Acetylcystein-aktivierten Kreatinkinase und dem Isoenzym MB bei Gesunden und bei Infarktpatienten berichtet.

\section{Methoden}

Für die Ermittlung des Normbereiches der Kreatinkinaseaktivität und die Etablierung der Entscheidungsgrenze für die Annahme einer signifikanten Kreatinkinase-MB-Aktivität wurden die Sera von 61 gesunden Personen ( 24 weiblich, 37 männlich) untersucht. Alle Probanden hatten normale Serumaktivitäten der Aspartat-

1) Merck Art. Nr. 14326. 
und Alanin-aminotransferase, $\gamma$-Glutamyltransferase und $\alpha$ Hydroxybutyratdehydrogenase sowie der Kreatinkinase (GSHaktiviert, (3)) und ihres Isoenzyms MB (GSH-aktiviert, (10)).

Bei 66 Patienten mit klinisch und elektrokardiographisch gesichertem, aktutem, transmuralem Myokardinfarkt (14) wurde die Aktivität der Kreatinkinase (359 Messungen, jeweils GSHund N-Acetylcystein-aktiviert) und der Kreatinkinase-MB (293 Messungen, jewveils GSH- und N-Acetylcystein-aktiviert) gemessen. Die Testpackungen für die Kreatinkinase (GSH-aktiviert) wurden von Boehringer Mannheim ${ }^{2}$ ), die Testpackungen für das Isoeñzym MB (GSH-aktiviert) ${ }^{3}$ ), (N-Acetylcy stein-aktiviert) ${ }^{4}$ ) und für die Kreatinkinase (N-Acetylcystein-aktiviert) ${ }^{5}$ ) wurden von E. Merck, Darmstadt, bezoger. Alle Tests enthielten über die dekJarierten Inhaltsstoffe hinaus EDTA als Aktivator.

Die Messungen der Kreatinkinase-Aktivitäten wurden entsprechend den Empfehlungen der Deutschen Gesellschaft für Klinische Chemie $(6,15)$ durchgeführt. Für die Messung der Kreatinkinase-MB-Aktivität wurde ein 1-Glas-Test (GSH-aktiviert) bzw. eine Methode mit Kreatinphosphat als Startreagenz (N-Acetylcystein-aktiviert) verwendet. Die Messungen wurden am Eppendorf-Enzymmeßplatz mit Küvettenwechselautomat, Enzymrechner und lin-log-Schreiber bei einer Meßtemperatur von $25^{\circ} \mathrm{C}$ durchgeführt. Für die Bestimmung des Reagenzienleerwertes wurden Sera 30 Minuten bei $56^{\circ} \mathrm{C}$ inaktiviert; nach Zugabe von Kreatinphosphat wurde die Aktivität im Ansatz gemessen. Die Linearität des Substratumsatzes wurde als gesichert angesehen, wenn graphisch und rechnerisch die Abweichungen unter 10\% lagen. Negative Kreatinkinase-MB-Aktivitäten bei der mit Glutathion aktivierten Methode wurden wegen der naheliegenden Verfälschung durch Glutathion-Reduktase in die Untersuchungen nicht miteinbezogen.

\section{Ergebnisse}

\section{Kreatinkinase - Normbereich}

Der Reagenzienleerwert für die Kreatinkinase (N-Acetylcystein-aktiviert) lag im Mittel bei 1,1 U/1 bei einer Standardabweichung von $1,5 \mathrm{U} / 1(\mathrm{n}=24)$. Bei $61 \mathrm{klinisch}$ und klinisch-chemisch gesunden Probanden jüngeren und mittleren Alters (17-58 Jahre) wurden die Aktivitäten der Kreatinkinase mit der GSH-aktivierten Methode und mit der N-Acetylcystein-aktivierten Methode bestimmt. Vor dem Start der Reaktion wurde für jedes Serum mit der N-Acetylcystein-aktivierten Methode der Probenleerwert ermittelt. Er betrug im Mittel 3,4 U/1 bei einer Standardabweichung von ebenfalls 3,4 U/1.

Der Mittelwert der Kreatinkinase (N-Acetylcystein-aktiviert) betrug unter der Annahme einer Normalverteilung 25,4 U/1 bei den gesunden Probanden, die Standardabweichung errechnete sich zu 11,0 U/1. Der in üblicher Weise definierte Normbereich $(\overline{\mathrm{x}} \pm 2,6$ Standardabweichungen) betrug daher für die Kreatinkinase ( $\mathrm{N}$-Acetyl cystein-aktiviert) 0-54 U/1 gegenüber 0-46 U/1 für die Kreatinkinase (GSH-aktiviert) bei unserem Kollektiv.

Die Korrelation zwischen Kreatinkinase (GSH-aktiviert) und'Kreatinkinase ( $\mathrm{N}$-Acetylcystein-aktiviert) betrug bei gesunden Personen $r=0,90$, die Regressionsrechnung ergab die in Abbildung 1 dargestellten Verhältnisse mit

\footnotetext{
2) Boehringer Mannheim Monotest Best. Nr. 124150.

3) Merck-1-Test CK-MB Best. Nr. 14300.

4) Merckotest CK NAC aktiviert Best. Nr. 14327.

5) Merckotest CK-MB NAC aktiviert Best. Nr. 14326.
}

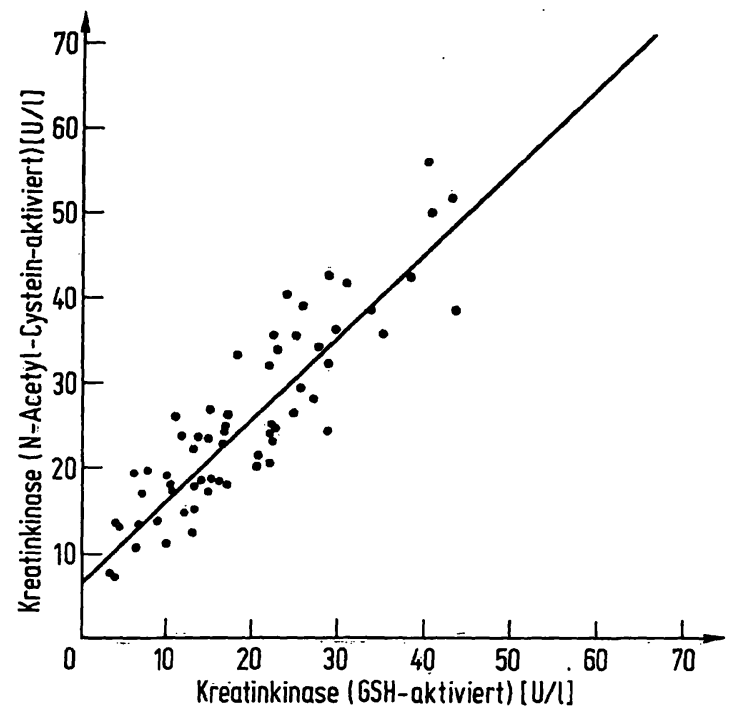

Abb. 1. Vergleich der Kreatinkinase (GSH-aktiviert (3)) und der Kreatinkinase (N-Acetylcystein-aktiviert (4)) bei 61 gesunden Personen. Korrelation und Regression. $\mathrm{n}=61, \mathrm{y}=0,95 \mathrm{x}+6,77, \mathrm{r}=0,90$

einem Regeressionsfaktor von 0,95 und einem konstanten Glied von $+6,77$ bei einem linearen Zusammenhang beider Meßgrößen.

\section{Kreatinkinase-MB - Entscheidungsgrenze}

Beim Gesunden werden keine meßbaren KreatinkinaseMB-Aktivitäten beobachtet. Durch die geringe Empfindlichkeit der Methode können bereits Schwankungen des Photostromes Enzymaktivitäten vortäuschen, ferner sind unspezifische Reaktionen vor Serumzugabe (Reagenzienleerwert) und nach Serumżugabe vor dem Start mit Kreatinphosphat zu berücksichtigen. Für die Praxis ist daher die Ermittlung einer Entscheidungsgrenze erforderlich, bei deren Überschreitung das Vorliegen einer signifikanten Isoenzymaktivität sehr wahrscheinlich ist.

Der Reagenzienleerwert für die Kreatinkinase-MB (NAcetylcystein-aktiviert) ergab sich bei Verwendung von inaktivem Serum nach Start der Reaktion mit Kreatinphosphat zu durchschnittlich $4,8 \mathrm{U} / 1$ bei einer Standardabweichung von $1,0 \mathrm{U} / \mathrm{l}(\mathrm{n}=24)$.

Bei 61 gesunden Probanden bestimmten wir den Probenleerwert mit der Kreatinkinase-MB (N-Acetylcystein-aktiviert) im Mittel zu 1,1 U/1 bei einer Standardabweichung von 2,0 U/1. Der als Kreatinkinase-MB-Aktivität gemèssene Substratumsatz bei unserem Normalkollektiv betrug unter der Annahme einer Normalverteilung im Durchschnitt 3,0 U/1, die Standardabweichung errechnete sich bei den 61 Personen unseres Kollektivs żü 2,0 U/1. Die Entscheidungsgrenze für die Annahme einer signifikanten Kreatinkinase-MB-Aktivität $(\overline{\mathrm{x}}+2,6 \mathrm{~s})$ lag daher bei einer Aktivität von $\geqslant 8,2 \mathrm{U} / 1$, bei Annahme einer nicht norma: len Verteilung $(\bar{x}+3.0 \mathrm{~s})$ bei einem Wert $\geqslant 9,0 \mathrm{U} / 1$. 
Eine Korrelations- und Regressionsrechnung zwischen Kreatinkinase-MB (GSH-aktiviert) und Kreatinkinase-MB (N-Acetylcystein-aktiviert) erschien nicht sinnvoll, da die als Kreatinkinase-MB-Aktivitäten gemessenen Werte mit großer Wahrscheinlichkeit keinem spezifischen Substratumsatz entsprechen.

\section{Kreatinkinase - Myokardinfarkt}

Die Beziehungen zwischen Kreatinkinase (GSH-aktiviert) und Kreatinkinase (N-Acetylcystein-aktiviert) wurden an einem Kollektiv von 66 Patienten mit akutem, transmuralem Myokardinfarkt ${ }^{6}$ ) durch 359 Parallelmessungen überprüft. Es ergab sich ein Korrelationskoeffizient von $r=0,97$. Die Regressionsgerade (Abb. 2) folgte der Gleichung $y=1,4 x+2,9$. Wie auch beim Normalkollektiv bestand ein linearer Zusammenhang; die mit N-Acetylcystein als Aktivator gemessenen Kréatinkinaseaktivitäten waren jedoch deutlich höher als die mit der GSH-Methode gefundenen Werte.

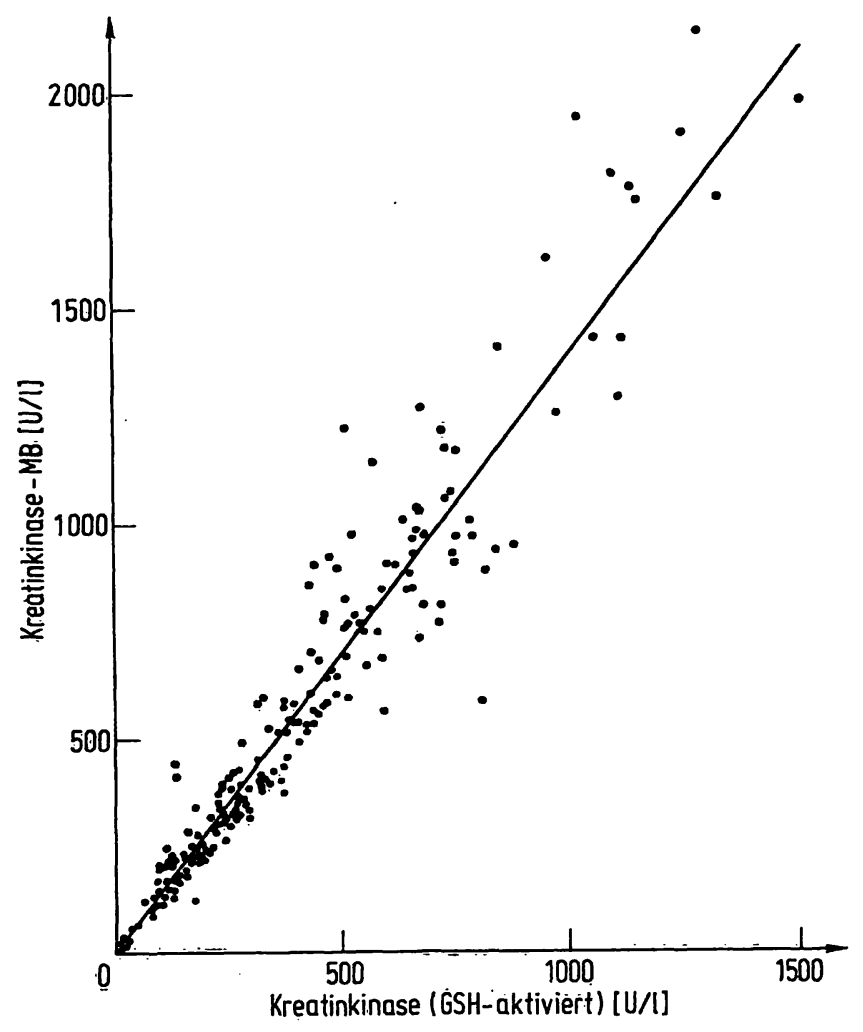

Abb. 2. Vergleich der Kreatinkinase (GSH-aktiviert (3)) und der Kreatinkinase (N-Acetylcystein=aktiviert (4)) bei $66 \mathrm{~Pa}-$ tienten mit akutem Myokardinfarkt. Korrelation und Regression bei 359 Wertepaaren. $\mathrm{n}=66,359$ Messungen, $\mathrm{y}=1,40 \mathrm{x}+2,90, \mathrm{I}=0,97$

\section{Kreatinkinase $=\mathrm{MB}$ - Myokardinfarkt}

Für die Beurteilung des Zusammenhanges zwișchen Kreatinkinase MB (GSH-aktiviert) und Kreatinkinase MB (NAcetylcystein-aktiviert) konnten 293 Parallelimessungen

6) Die hier mitgeteilten Dạten sind Teil einer Inauguraldissertation (1).

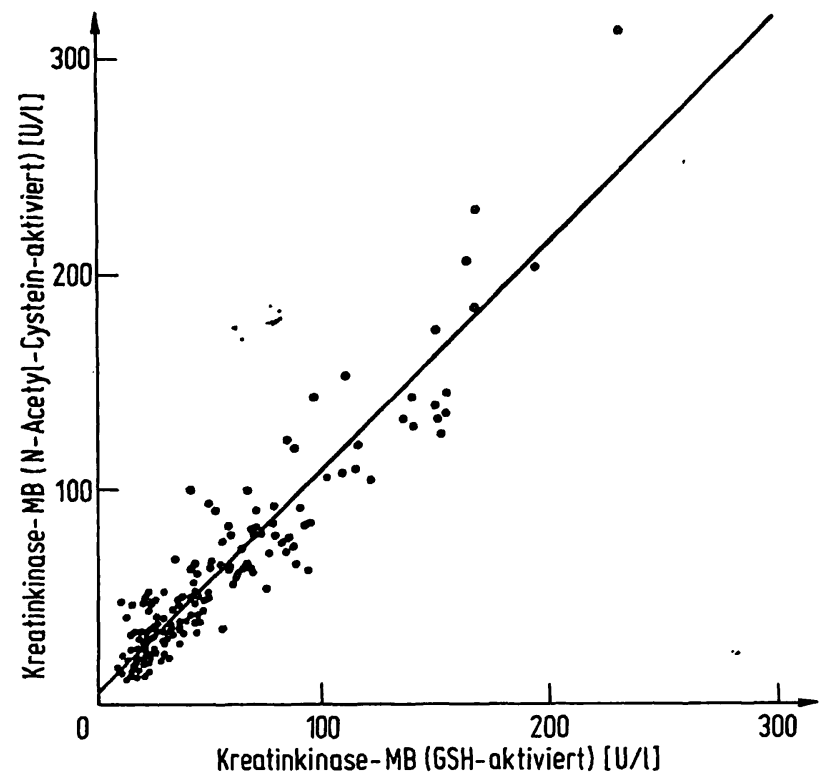

Abb. 3. Vergleich der Kreatinkinase-MB (GSH-aktiviert (3)) und der Kreatinkinase-MB (N-Acetylcystein-aktiviert (4)) bei 66 Patienten mit akutem Myokardinfarkt. Korrelation und Regression bei 293 Wertepaaren.

$n=66,293$ Messungen, $y=1,05 x+4,94, r=0,96$

an den Sera unserer 66 Patienten mit akutem Myokardinfarkt herangezogen werden. Der Korrelationskoeffizient betrug $r=0,96$; $z$ wischen beiden Parametern bestand ein linearer Zusammenhang (Abb. 3), der sich durch die Regressionsgleichung $y=1,1 x+4,9$ beschreiben ließ.

\section{Relative Kreatinkinase-MB - Myokardinfarkt}

Durch die Berechnung der relativen Kreatinkinase-MBAktivität, bezogen auf die Gesamtkreatinkinaseaktivität, kann die Spezifität dieses Isoenzyms verbessert werden. Bei 65 unserer Patienten ermittelten wir unter Verwendung von 228 Meßwertpaaren für die Kreatinkinase (NAcetylcystein-aktiviert) und die Kreatinkinase-MB (NAcetylcystein-aktiviert) eine log-normale Verteilung der relativen Kreatinkinase-MB-Aktivitäten mit einem Mittelwert von $10 \%$ der Gesamtaktivität. Die Trenngrenze für die Annahme einer extrakardial bedingten KreatinkinaseMB-Aktivität ergab sich für unser Kollektiv damit zu ( $\bar{x}-$ 2,6 s) $\leqslant 5,7 \%$ der Gesamtaktivität.

\section{Diskussion}

Kreatinkinase - Normbereich

Durch die Verwendung von N-Acetylcystein und den $\mathrm{Zu}$ satz vom AMP und Diadenosin-pentaphosphat bei der neuen Kreatinkinase-Standardmethode (6) werden Meßfehler durch Glutathion-Reduktase und Adenylatkinase aus Erythrocyten weitgehend ausgeschlossen $(2,6,7)$. Für praktische Belange war die Frage zu untersuchen, inwieweit die Änderung der Methode von einer Änderung der Normbereiche begleitet wird. Da für eine Über- 
gangsperiode beide Methoden nebeneinander benutzt werden dürten, war ferner zu entscheiden, ob im Einzelfall Kreatinkinase-Aktivitäten (GSH-aktiviert) in Kreatinkinase-Aktivitäten (N-Acetylcystein-aktiviert) durch Einführung eines Korrekturfaktors $(16,17)$ umgerechnet werden dürfen. Dabei ergeben sich hinsichtlich des Reagenzienleerwertes keine signifikanten Unterschiede, wie ein Vergleich unserer Daten (0-5,4 U/1) mit den Angaben für die Kreatinkinase (GSH-aktiviert, Methode mit Serumstart) zeigt (18).

Wie die an unserem Kollektiv von gesunden Probanden beiderlei Geschlechts ermittelten Daten zeigen, unterscheidet sich der obere Grenzwert der GSH-aktivierten Methode um $10 \mathrm{U} / 1$ von der N-Acetylcystein-aktivierten Methode; da die Steigung der Regressionsgeraden nahezu 1 beträgt, sind die geringfügig höheren Aktivitäten der Kreatinkinase (N-Acetylcystein-aktiviert) zum größten Teil Ausdruck des absoluten Gliedes in der Regressionsgleichung.

Unsere Ergebnisse entsprechen in zweierlei Hinsicht nicht ganz den bisher in der Literatur mitgeteilten Befunden. Der obere Grenzwert fur die Kreatinkinase (N-Acetylcystein-aktiviert) wurde bisher mit $60 \mathrm{U} / 1$ (Frauen) bzw. $70 \mathrm{U} / 1$ (Männer) angegeben $(6,19,20)$. Mit rund $55 \mathrm{U} / 1$ bei einem gemischten Kollektiv liegt die von uns ermittelte Normgrenze etwas niedriger.

Bisher wurde davon ausgegangen, daß die Verwendung von $\mathrm{N}$-Acetylcystein die Empfindlichkeit der Kreatinkinasebestimmung um den Faktor 1,5 steigert $(6,16,17)$. Bei ausschließlicher Verwendung frischer Serumproben bei unserem Normalkollektiv fanden wir lediglich eine Absolutdifferenz von etwa $7 \mathrm{U} / 1$.

Es ist daher anzunehmen, daß die von uns nachgewiesene, vergleichbare Empfindlichkeit auf der Untersuchung voll aktiver Sera beruht. Vorläufige Ergebnisse von Serumalterungsversuchen scheinen diese These zu belegen. Bei der Besprechung der Ergebnisse bei Infarktpatienten wird auf das Problem noch weiter eingegangen. Eine Umrechnung von Kreatinkinase-Aktivitäten GSH-aktiviert in $\mathrm{N}$ Acetylcystein-aktiviert und vice versa ist nach unseren Befunden jedenfalls problematisch.

\section{Kreatinkinase-MB - Entscheidungsgrenze}

Ebenso wie bei der Kreatinkinase lagen die Reagenzienleerwerte bei der N-Acetylcystein-aktivierten Kreatinkinase-MB in dem für die GSH-aktivierte Methode mitgeteilten Bereich (17). Die Entscheidungsgrenze für die Annahme einer signifikanten Kreatinkinase-MB-Aktivität (N-Acetylcystein-aktiviert) ermittelten wir zu 8-9 U/1. Höhere Aktivitäten entsprechen einer pathologischen Kreatinkinase-MB-Aktivität. Dieser Grenzwert stimmt mit den für die Kreatinkinase-MB (GSH-aktiviert) mitgeteilten Befunden $(10,13)$, die inzwischen vielfach überprüft wurden $(9,17,21-25)$, überein. Mehr noch als bei der Gesamtkreatinkinase ergibt sich gegenüber der GSHaktivierte Methode keine Änderung in der Bewertung der Grenzaktivität. Vorteilhaft erscheint bei der N-Acetylcystein-aktivierten Kreatinkinase-MB, daß nach der vorgeschriebenen Inkubationszeit der Reaktionsverlauf regelmäßig linear war.

\section{Kreatinkinase - Myokardinfarkt}

Im Unterschied zu den Messungen an gesunden Probanden wurden die Sera von Patienten mit akutem, transmuralem Myokardinfarkt teilweise erst 5-6 Stunden nạch der Probenahme untersucht. Dabei ergaben sich mit der Kreatinkinase (N-Acetylcystein-aktiviert) im Durchschnitt um den Faktor 1,4 höhere Aktivitäten als mit der GSH-aktivierten Methode. Dieser Wert stimmt mit den vorliegenden Literaturangaben überein $(6,16,26)$.

\section{Kreatinkinase-MB - Myokardinfarkt}

Die Ergebnisse beim Vergleich der Kreatinkinase-MB-Aktivität nach Messung mit GSH und N-Acetylcystein als Aktivator bei Myokardinfarkt ergeben ähṇliche Verhältnisse wie bei dem Normalkollektiv. Im Durchschnitt unterscheiden sich die Enzymaktivitäten wiederum nur durch einen festen Betrag von etwa 5 U/1, die Steigerung der Regressionsgeraden liegt nahe bei 1 , keinesfalls aber in dem für die Kreatinkinase gefundenen und bisher beschriebenen Bereich von $1,5-2,0(6,17)$.

Eine denkbare Erklärung für diese Beobachtung sehen wir in der Annahme, daß es analog zu den Verhältnișsen bei Verwendung von Dithiothreit zu einer uniformen Aktivierung des Isoenzyms MB unabhängig vom verwendeten Aktivator nach Ausschaltung der Kreatinkinase-MM durch Immuninhibition kommt (27). Die bei der Gesamtkreatinkinase in Abhängigkeit vom eingesetzten Sulfhydrylreagenz gefundene Differenz der Aktivitäten könnte aber auch auf eine unterschiedliche Empfindlichkeit von Kreatinkinase-MM zurückzuführen sein, während die Kreatinkinase-MB unabhängig vom verwendeten Aktivator reaktiviert wird.

\section{Relative Kreatinkinase-M B - Myokardinfarkt}

Aus 228 Kreatinkinase-MB: Kreatinkinse-Quotienten ermittelten wir für die Annahme einer extrákardialen Herkunft einer Isoenzymaktivität eine Trenngrenze von rund 6\%. Dieser Prozentsatz entspricht früheren Mitteilungen für die Kreatinkinase-MB (GSH-aktiviert) $(9,22,25,26)$, obwohl eigene Untersuchungen einen Trennwert von $7 \%$ ergaben (1). Da die Gesamtkreatinkinaseaktivitäten (NAcetylcystein-aktiviërt) in unserem Infárktkollektiv im Mittel um den Faktor 1,4 höher lagen als bei der GSHaktiviert Methode, während die Kreatinkinase-MB-Aktivitäten sich als' weitgehend unabhängig vom verwendeten Aktivator erwiesen, würde man auch einen niedrigeren Trennwert für die relative Kreatinkinạse MB (N-Acetylcystein-aktiviert) erwarten. 
Zusammenfassend läßt sich sagen, daß durch die Einführung der neuen Optimierten Standardmethode die Normgrenze für die Kreatinkinaseaktivität nur unwesent- lich erhöht wurde, während sich für die Kreatinkinase-MB gegenüber dem bisherigen Verfahren keine Änderung ergibt.

\section{Literatur}

1. Schmidt, E. W., W. Bender \& A. Wellstein: In Vorbereitung.

2. Szasz, G. (1975), in: Proceedings of the Second International Symposium on Clinical Enzymology (N. W. Tietz, A. Wennstock, D. O. Rodgerson eds.), AACC, Winston-Salem, S. 143-179.

3. Szasz, G., W. Gruber \& E. Bernt (1976) Clin. Chem., 22, 650-656.

4. Weidemann, G. (1973), J. Clin. Chem. Clin. Biochem., 11, $134-135$.

5. Weidemann, G. \& R. D. Schmidt (1978), J. Clin. Chem. Clin. Biochen., 16, 253-254.

6. Dtsch. Ges. f. Klin. Chemie (1977), J. Clin. Chem. Clin. Biochen., 15, 249-254.

7. Szasz, G., W. Gerhardt, W. Gruber \& E. Bernt (1976), Clin. Chem. 22, 1806-1811.

8. Blume, H., E. W. Schmidt, S. Caquela, H. Ostheimer, V. Perge, R. Stankovic \& A. Wellstein (1978), Anaesthesist, 27108-114.

9. Neumeier, D., B. Kemkes, B. Glück \& M. Knedel (1977), J. Clin. Chem. Clin. Biochem., 15, 179.

10. Prellwitz, W., D. Neumeier, M. Knedel, H. Lang, H. Würzburg, H. Schönborn \& H.-P. Schuster: (1976), Dtsch. Med. Wochenschr., 101, 983-988.

11. Schmidt, E. W., A. Wellstein \& A. Moll (1977), Med. Klin., $72,1368-1371$.

12. Wellstein, A. \& E. W. Schmidt (1977), Ärztl. Lab. 23, 334336.

13. Würzburg, H., N. Hennrich, H. Lang, W. Prellwitz, D. Neumeier \& M. Knedel (1976), Klin. Wochenschr., 54, 357-360.
14. Bender, W.: Inauguraldissertation, Mainz in Vorbereitung.

15. Dtsch. Ges. f. Klin. Chemie (1972), J. Clin. Chem. Clin. Biochem., 10, 182-192.

16. Lang, H. (1977), Pers. Mitt.

17. Würzburg, H. \& W. Prellwitz (1977), J. Clin. Chem. Clin. Biochem., 15, 198.

18. Szasz, G., E. W. Busch \& H. B. Farohs (1970), Dtsch. Med. Wochenschr., 95, 829-835.

19. Szasz, G. \& E. W. Busch (1975), Clin. Chem., 21, 1008.

20. Szasz, G. (1975), in: 1. c. (2).

21. Klapdor, R., K. Harm \& G. Kugler (1977), Dtsch. Med. Wochenschr., 102, 1309-1314.

22. Klapdor, R., K. Harm \& A. Müller-Jensen (1977), Herz-Kreisl., 9, 761-763.

23. Neumeier, D., W. Prellwitz, H. Würzburg, M. Brundobler, M. Olbermann, H.-J. Just, M. Knedel \& H. Lang (1976), Clin. Chim. Acta, 73, 445-451.

24. Prellwitz, W. \& D. Neumeier (1976), Internist, 17, 436451.

25. Prellwitz, W., S. Kapp, D. Neumeier, M. Knedel, H. Lang \& D. Henwinkel (1978), Klin. Wochenschr., 56, 559-565.

26. Wellstein, A.: (1977), Inauguraldissertation, Mainz.

27. Rao, P. S., J. J. Lucas, S. M. Agres \& H. Mudler (1975), Clin. Chem., 21, 1612-1618.

Dr. Ernst W. Schmidt Chefarzt des Instituts für Laboratoriumsmedizin Stadtkrankenhaus D-6090 Rüsselsheim 


$$
\text { . }
$$

